\title{
An Improved Succinct Representation for Dynamic $k$-ary Trees
}

\author{
Diego Arroyuelo* \\ Dept. of Computer Science, University of Chile, \\ darroyue@dcc.uchile.cl
}

\begin{abstract}
. $k$-ary trees are a fundamental data structure in many textprocessing algorithms (e.g., text searching). The traditional pointer-based representation of trees is space consuming, and hence only relatively small trees can be kept in main memory. Nowadays, however, many applications need to store a huge amount of information. In this paper we present a succinct representation for dynamic $k$-ary trees of $n$ nodes, requiring $2 n+n \log k+o(n \log k)$ bits of space, which is close to the information-theoretic lower bound. Unlike alternative representations where the operations on the tree can be usually computed in $O(\log n)$ time, our data structure is able to take advantage of asymptotically smaller values of $k$, supporting the basic operations parent and child in $O(\log k+\log \log n)$ time, which is $o(\log n)$ time whenever $\log k=$ $o(\log n)$. Insertions and deletions of leaves in the tree are supported in $O\left((\log k+\log \log n)\left(1+\frac{\log k}{\log (\log k+\log \log n)}\right)\right)$ amortized time. Our representation also supports more specialized operations (like subtreesize, depth, etc.), and provides a new trade-off when $k=O(1)$ allowing faster updates (in $O(\log \log n)$ amortized time, versus the amortized time of $O\left((\log \log n)^{1+\epsilon}\right)$, for $\epsilon>0$, from Raman and Rao [21]), at the cost of slower basic operations (in $O(\log \log n)$ time, versus $O(1)$ time of [21]).
\end{abstract}

\section{Introduction and Previous Works}

In this paper we study the problem of the succinct representation of dynamic $k$-ary trees, or cardinal trees, or simply tries, i.e. trees such that the children of a node are sorted and labeled with a symbol drawn from the alphabet $\{1, \ldots, k\}$. We assume that $k$ is fixed, yet we note it as a variable in our analysis: think for example of a trie representing information about a text on an (large) alphabet.

A succinct data structure requires space close to the information-theoretic lower bound (besides lower-order additive terms). Since the number of different $k$-ary trees with $n$ nodes is $\frac{1}{k n+1}\left(\begin{array}{c}k n+1 \\ n\end{array}\right)$, the information-theoretical lower bound for the number of bits to represent a $k$-ary tree is $C(n, k)=\log \left(\frac{1}{k n+1}\left(\begin{array}{c}k n+1 \\ n\end{array}\right)\right)$ ( $\log x$ means $\left\lceil\log _{2} x\right\rceil$ in this paper) which, assuming that $k$ is a function of $n$, is $C(n, k) \approx 2 n+n \log k-o(n+\log k)$ bits. In most succinct representations of $k$-ary trees, the first term stands for the encoding of the tree structure, and the second term for the space required to code the labels of the edges.

\footnotetext{
* Supported in part by Yahoo! Research Latin America and Fondecyt Grant 1-080019.
} 
Besides requiring little space, succinct data structures in general support operations as efficiently as their non-space-efficient counterparts. In the case of trees, we are interested in succinct representations that can be navigated in the usual way, as many compact representations cannot be navigated [15]. We are interested in the following operations: parent $(x)$, which gets the parent of node $x$; $\operatorname{child}(x, i)$, which gets the $i$-th child of node $x$; $\operatorname{child}(x, \alpha)$, which gets the child of node $x$ labeled by symbol $\alpha \in\{1, \ldots, k\}$; depth $(x)$, which gets the depth of node $x$ in the tree; degree $(x)$, which gets the number of children of node $x$; subtreesize $(x)$, which gets the size of the subtree of node $x$; preorder $(x)$, which gets the preorder number of node $x$; and isancestor $(x, y)$, which tells us whether node $x$ is an ancestor of node $y$. In the context of dynamic trees, the representation should support operations insert and delete as well, which respectively allow us to add new nodes and delete existing nodes from the tree.

We consider the standard word RAM model of computation, in which every word of size $w=\Theta(\log n)$ bits can be accessed in constant time. Basic arithmetic and logical operations can be computed in constant time. Unless stated, we will assume that all navigations start from the root of the tree, and that insertions and deletions occur at leaves, which is usual in many applications [19,21].

The pointer-based representation of a tree requires $O(n \log n)$ bits for the tree structure, which is space consuming and hence only small trees can be kept in a fast memory. Typical examples where this matters are that of DOM trees for XML documents, and suffix trees [1] for full-text search applications.

Starting with the work of Jacobson [13], a number of succinct representations have been defined for static trees $[18,5,20,10,14,8]$, each providing a different set of operations and complexities. The case of succinct representation of dynamic trees has been studied only for binary trees by Munro et al.[19] and Raman and Rao [21]; the latter work provides a representation requiring $2 n+o(n)$ bits, and supports basic navigation operations in worst-case constant time, while updates in the tree can be performed in $O\left((\log \log n)^{1+\epsilon}\right)$ amortized time, for any constant $\epsilon>0$. The efficient representation of succinct dynamic $k$-ary trees was posed as an open problem by Munro et al. [19], as adapting these representations for dynamic $k$-ary trees by transforming the tree into binary gives poor results: basic navigation operations like parent and child now become $O(k)$ in the worst case, which is not so advantageous if $k$ is not a constant, as in many applications.

Another alternative to represent a dynamic $k$-ary tree succinctly is to use the dynamic data structure for balanced parentheses of Chan et al. [7] to represent the Depth-First Unary Degree Sequence (DFUDS) [5] of the tree. Thus, the basic update and navigation operations are supported basically in $O(\log n)$ time; however, the time complexity of those operations depends on $n$, the number of nodes in the tree, rather than on $k$, the alphabet size. Then, this structure cannot take advantage of asymptotically smaller values of $k$.

In this paper we present an improved succinct representation for dynamic $k$-ary trees, which is faster than both Raman and Rao [21] and Chan et al. [7]'s solutions. Our data structure requires $2 n+n \log k+o(n \log k)$ bits of space, and supports operations parent $(x)$ and $\operatorname{child}(x, i)$ in $O(\log k+\log \log n)$ worst-case 
time, which is $o(\log n)$ whenever $\log k=o(\log n)$ holds. Updates are supported in $O\left((\log k+\log \log n)\left(1+\frac{\log k}{\log (\log k+\log \log n)}\right)\right)$ amortized time. We are also able to compute operation $\operatorname{child}(x, \alpha)$ in $O\left((\log k+\log \log n)\left(1+\frac{\log k}{\log (\log k+\log \log n)}\right)\right)$ worst-case time. For the particular case where $k=O(1)$ (for example, binary trees), our representation provides a new trade-off, supporting basic operations in $O(\log \log n)$ time (versus $O(1)$ time of [21]) and updates in $O(\log \log n)$ amortized time (versus $O\left((\log \log n)^{1+\epsilon}\right)$ amortized time of [21], for any constant $\left.\epsilon>0\right)$.

Our basic approach to solve the problem is similar to existing approaches $[19,21]$ : we divide the tree into small blocks which are easier to update upon tree modifications. However, in the case of $k$-ary trees we have to face additional problems: structuring inside blocks, defining the adequate block size in order to require $o(n)$ bits for the inter-block pointers, supporting block overflows (which cannot be carried out by using table lookups [19,21], since our blocks are large enough so as to be used to index a table), etc.

Besides the fact that dynamic $k$-ary trees is a fundamental data structure, our work is also motivated by previous works [3,2] on compressed full-text indexes, in particular Lempel-Ziv compressed indexes. The results of this paper will help us to improve the construction time of Lempel-Ziv indexes [3], and will be a base to define a dynamic compressed full-text index on that of [2].

\section{Preliminary Concepts}

Data Structures for rank and select Queries. Given a sequence $S[1 . . n]$ over an alphabet $\{1, \ldots, k\}$ and given any $c \in\{1, \ldots, k\}$, we define operation $\operatorname{rank}_{c}(S, i)$ as the number of $c$ s up to position $i$ of $S$. Operation $\operatorname{select}_{c}(S, j)$ yields the position of the $j$-th $c$ in $S$. In the dynamic case we also want to insert/delete symbols into/from the sequence. The data structure of González and Navarro [11] supports all the operations (including insert and delete) in $O\left(\log n\left(1+\frac{\log k}{\log \log n}\right)\right)$ worst-case time, requiring $n H_{0}(S)+o(n \log k)$ bits of space, where $H_{0}(S)$ denotes the 0 -th order empirical entropy of $S$ [17].

Data Structures for Searchable Partial Sums. Throughout this paper we will need data structures for searchable partial sums [12]. Given an array $A\left[1 . . n^{\prime}\right]$ of $n^{\prime}$ integers, these data structures allow one to retrieve $A[i]$ and support operations $\operatorname{Sum}(A, i)$, which computes $\sum_{j=1}^{i} A[j] ; \operatorname{Search}(A, i)$, which finds the smallest $j^{\prime}$ such that $\operatorname{Sum}\left(A, j^{\prime}\right) \geqslant i$; Update $(A, i, \delta)$, which sets $A[i] \leftarrow A[i]+\delta$; Insert $(A, i, e)$, which adds a new element $e$ to the set between elements $A[i-1]$ and $A[i]$; and Delete $(A, j)$, which deletes element $A[j]$.

Such an ADT is traditionally implemented through a red-black tree $T_{A}$ such that the leaves of the tree are the elements of $A$. Each internal node of $T_{A}$ stores a parent pointer, one bit indicating whether the node is the left or right child of its parent, the number $n l$ of elements (leaves) in the left subtree, and the sum $s l$ of the values in the leaves of its left subtree. The space required is $O\left(n^{\prime} \log n^{\prime}\right)$ bits. To compute the operations we navigate the tree using the information stored 
in each node. The tree can be also navigated from leaves to root, in order to compute the sum up to a given position in $A$ (leaf of $T_{A}$ ) without necessarily knowing the position $j^{\prime}$, but just the corresponding leaf of $T_{A}$. Thus, all the operations can be supported in $O\left(\log n^{\prime}\right)$ time.

Data Structures for Balanced Parentheses. Given a sequence $P$ of $2 n$ balanced parentheses, we want to support the following operations. findclose $(P, i)$ : given an opening parenthesis at position $i$, finds the matching closing parenthesis; findopen $(P, j)$ : given a closing parenthesis at position $j$, finds the matching opening parenthesis; excess $(P, i)$ : yields the difference between the number of opening and closing parenthesis up to position $i$ in $P$; enclose $(P, i)$ : given a parenthesis pair whose opening parenthesis is at position $i$, yields the opening parenthesis corresponding to the closest matching pair enclosing $i$.

Munro and Raman [18] showed how to implement all these operations in constant time and using $2 n+o(n)$ bits. They also showed one of the applications of sequences of balanced parentheses: the succinct representation of general trees.

In the dynamic case, the parentheses sequence can change over time, by inserting/deleting a new pair of matching parenthesis into/from the sequence. The data structure of Chan et al. [7] supports all of the operations, including insertions and deletions, in $O(\log n)$ time and requires $O(n)$ bits of space. The space can be dropped to $2 n+o(n)$ bits of space if we represent the parentheses as a binary string of length $2 n$, which is then represented using the dynamic data structure of Mäkinen and Navarro [16], requiring overall $2 n+o(n)$ bits and supporting rank and select on the parentheses in $O(\log n)$ worst-case time, as well as the insertion and deletion of new elements in $O(\log n)$ worst-case time. Since both solutions are similar, we add the extra index of [7] to this data structure in order to be able to compute the parenthesis operations, including updates, in $O(\log n)$ worst-case time and using $o(n)$ extra bits of space.

Lemma 1. There exists a representation for a dynamic sequence of $2 N$ balanced parentheses using $2 N+o(N)$ bits of space and supporting operations findclose, findopen, excess, enclose, rank, select, insert, and delete, all of them in $O(\log N)$ worst-case time.

Succinct Representation of Trees. There are a number of succinct representations of static trees, such as LOUDS [13], balanced parentheses [18], DFUDS [5], ordinal trees [10], and the ultra succinct representation of [14], requiring $2 n+o(n)$ bits and allowing different sets of operations.

In particular, the DFUDS [5] representation supports all of the static-tree operations, including $\operatorname{child}(x, i)$, $\operatorname{child}(x, \alpha)$, degree, and depth (the latter using the approach of Jansson et al. [14]) in constant time. To get this representation [5] we perform a preorder traversal on the tree, and for every node reached we write its degree in unary using parentheses. What we get is almost a balanced parentheses representation: we only need to add a fictitious ' (' at the beginning of the sequence. A node of degree $d$ is identified by the position of the first of the $d+1$ parentheses representing the node. 
In order to support $\operatorname{child}(x, \alpha)$ on the DFUDS representation we store the sequence $S$ of edge labels according to a DFUDS traversal of the tree. In this way, the labels of the children of a given node are all stored contiguously in $S$. We represent $S$ with a data structure for rank and select [9]. Let $p$ be the position of node $x$ within the DFUDS sequence $D$, and let $p^{\prime}=\operatorname{rank}_{(}(D, p)$ be the position in $S$ of the symbol for the first child of $x$. Let $n_{\alpha}=\operatorname{rank}_{\alpha}\left(S, p^{\prime}-1\right)$ and $i=\operatorname{select}_{\alpha}\left(S, n_{\alpha}+1\right)$. If $i$ lies within positions $p^{\prime}$ and $p^{\prime}+\operatorname{degree}(x)-1$, the child we are looking for is the $\left(i-p^{\prime}+1\right)$-th child of $x$, which is computed in constant time as child $\left(x, i-p^{\prime}+1\right)$; otherwise $x$ has not a child labeled $\alpha$. This approach, which is also used by Barbay et al. [4], is different to the original one [5], yet ours is easier to be dynamized by using the data structures of [11].

For succinct dynamic binary trees, the representation of Munro et. al. [19] requires $2 n+o(n)$ bits, and they allow updates in $O\left(\log ^{2} n\right)$ amortized time, operations parent and child in $O(1)$ time, and subtreesize in $O\left(\log ^{2} n\right)$ time. Raman and Rao [21] improve the update time to $O\left((\log \log n)^{1+\epsilon}\right)$ amortized. More specialized operations are also supported in constant time.

\section{$3 \quad$ Improved Succinct Dynamic $k$-ary Trees}

Given a static succinct representation of a tree, as for example DFUDs, if we want to insert a new node at any position in the tree, we must rebuild the corresponding sequence from scratch. The methods for succinct representation of dynamic binary trees $[19,21]$ can be adapted to represent dynamic $k$-ary trees by transforming the tree into a binary one. However, the cost of basic navigation operations such as parent and child now becomes $O(k)$ in the worst case, while the insertion cost remains the same, $O\left((\log \log n)^{1+\epsilon}\right)$ amortized time.

Another alternative is to represent the DFUDS of the $k$-ary tree with the data structure of Lemma 1, and the edge labels by a dynamic data structure for rank and select [11]. Thus, the basic navigation operations are supported in $O(\log n)$ time $\left(\operatorname{child}(x, \alpha)\right.$ is supported in $O\left(\log n\left(1+\frac{\log k}{\log \log n}\right)\right)$ time if we use [11]). The overall space requirement is $2 n+n \log k+o(n \log k)$ bits. Notice that the time complexity of these navigation operations is related to $n$, the number of nodes in the tree, rather than to $k$, the alphabet size. Thus, this data structure cannot take advantage of asymptotically smaller values of $k$, e.g. $k=O(\operatorname{polylog}(n))$.

In this section we present a succinct representation for dynamic $k$-ary trees, which is faster both than representing the $k$-ary tree using a data structure for dynamic binary trees [19,21] and than representing DFUDS with the data structure of Lemma 1 whenever $\log k=o(\log n)$ holds.

\subsection{Basic Tree Representation}

To allow efficient navigation and modification of the tree, we incrementally divide it into disjoint blocks, as in previous approaches [3,19,21]. Every block represents a connected component of $N$ nodes of the whole tree, such that $N_{\min } \leqslant N \leqslant N_{\max }$, for given minimum and maximum block sizes $N_{\min }$ and 
$N_{\text {max }}$, respectively. We arrange these blocks in a tree by adding inter-block pointers, and thus the entire tree is represented by a tree of connected components.

For each internal node $x$ of the tree there are two cases: node $x$ is internal to a block $p$ or $x$ is a leaf of block $p$ (but not a leaf of the whole tree). The latter nodes form what we call the frontier of a block, and every such node $x$ stores an inter-block pointer to a child block $q$ where the representation of the subtree of node $x$ starts. We duplicate node $x$ by storing it as a fictitious root of $q$, such that every block is a tree by itself. Therefore, every such node $x$ in the frontier of a block $p$ has two representations: $(\mathbf{1})$ as a leaf in block $p ;(\mathbf{2})$ as the root node of the child block $q$. Note that the former point enforces that sibling nodes are all stored in the same block, and the latter point enforces that every node is stored in the same block as its children. This property will be useful to simplify the navigation on the tree, as well as ensuring that a block can always be partitioned in the right way upon block overflows.

We have reduced the size of the problem, as insertions (or deletions) only need to update the block where the insertion is carried out, and not the whole tree. As every block is a tree by itself, we can represent them by using any succinct tree representation, which makes this representation very flexible. We can use DFUDS [5] to get constant-time navigation inside a block. Yet, this is a static representation, and so the update time would be linear in the block size.

Defining Block Sizes. Let us now define the values $N_{\min }$ and $N_{\max }$, the minimum and maximun block size respectively. Since inter-block pointers should require $o(n)$ bits overall, a pointer of $O(\log n)$ bits must point to a block of size $\Omega\left(\log ^{2} n\right)$ nodes, and therefore $N_{\min }=\Theta\left(\log ^{2} n\right)$. On the other hand, a block $p$ should have room to store at least the potential $k$ children of the root of the block (recall that sibling nodes must be stored all in the same block). Also, we must define $N_{\max }$ in such a way that when we insert a node in a block of maximal size $N_{\max }$ (i.e., the block overflows), we can split the block into two blocks, each of size at least $N_{\min }$. By defining $N_{\max }=\Theta\left(k \log ^{2} n\right)$, in the worst case (i.e., the case where the new created block has the smallest possible size) the root of the block has its $k$ possible children, the subtree of each such child having $\Theta\left(\log ^{2} n\right)$ nodes. Thus, upon an overflow, any of such subtrees of size at least $N_{\min }$ can be copied to a new block, requiring overall $o(n)$ bits for the pointers.

Existing related works $[3,19,21]$ use a static block representation, which is rebuilt from scratch upon insertions or deletions. In the case of binary trees $[19,21]$, these total reconstructions are carried out in constant time by using precomputed tables. However, for $k$-ary trees the blocks are large enough so as to be used to index a table. Thus, we use a different approach: we first reduce the size of the problem by updating smaller subtrees (the blocks), and then we make these smaller subtrees dynamic to avoid the linear update time.

Let us study now the block layout. Every block $p$ of $N$ nodes, having $N_{c}$ child blocks and root node $r_{p}$ is represented by: the tree topology of the block; a set of $N_{c}$ pointers $P T R_{p}$ to child blocks; a set of flags $F_{p}$ indicating the nodes in the frontier of $p$; and a pointer to the representation of $r_{p}$ in the parent block. 
Representing the Tree Topology of Blocks. We represent the tree structure $T_{p}$ of block $p$ plus the edge labels $S_{p}$ by using suitable dynamic data structures, to avoid rebuilding them from scratch upon updates.

The tree structure $T_{p}$ of each block $p$ is represented by the following data structure, where operation selectnode $(j)$ yields the DFUDS position of the node with preorder $j$ inside block $p$. (From now on we use the subscript $p$ to indicate operations local to a block $p$, i.e., disregarding the inter-block structure.)

Lemma 2. There exists a dynamic DFUDS representation for a tree $T_{p}$ of $N$ nodes requiring $2 N+o(N)$ bits of space and allowing us to compute operations parent ${ }_{p}$, child $(x, i)$, degree ${ }_{p}$, subtreesize ${ }_{p}, \operatorname{preorder}_{p}$, selectnode $_{p}$, insert ${ }_{p}$, and delete $_{p}$, all of them in $O(\log N)$ worst-case time.

Proof. We represent the DFUDS sequence of $T_{p}$ using the data structure of Lemma 1 , requiring $2 N+o(N)$ bits of space. Except for insert ${ }_{p}$ and delete ${ }_{p}$, operations on $T_{p}$ can be computed as defined originally in [5] for the static case, using the operations provided in Lemma 1 as a base, which take $O(\log N)$ time.

For operation insert $_{p}$, notice that the insertion of a new leaf $x$ will increase the degree of its parent node $y$ in the tree; this increase is carried out by adding a new opening parenthesis at the corresponding position within the representation of $y$. To represent the new leaf node, on the other hand, we must add a new closing parenthesis at the corresponding position within $T_{p}$. We can show that this new pair of opening and closing parentheses is a matching pair, and hence the insertion can be handled by the data structure of Lemma 1. Deletions are handled in a similar way. (Further details are deferred to the full paper.)

The overall space requirement of the tree structure for all the blocks is $2 n+$ $o(n)$ bits. In our case $N=O\left(k \log ^{2} n\right)$, so the operations on $T_{p}$ can be supported in $O(\log k+\log \log n)$ worst-case time.

We store the symbols labeling the edges of $T_{p}$ in array $S_{p}$, sorted according to DFUDS as in Section 2. We preprocess $S_{p}$ with a dynamic data structure for $\operatorname{rank}_{\alpha}\left(S_{p}, i\right)$ and $\operatorname{select}_{\alpha}\left(S_{p}, j\right)$ queries [11]. We can now compute operation child $_{p}(x, \alpha)$ inside block $p$ using the operations provided by the representation of $T_{p}$ (i.e., $\left.\operatorname{child}_{p}(x, i)\right)$ and that of $S_{p}$ (i.e., rank rand $_{\alpha}$ and select $\alpha$ ), as in Section 2, in $O\left(\log N\left(1+\frac{\log k}{\log \log N}\right)\right)=O\left((\log k+\log \log n)\left(1+\frac{\log k}{\log (\log k+\log \log n)}\right)\right)$ worst-case time. The insertion/deletion of a symbol to/from $S_{p}$ can be carried out within the same time complexity [11]. The space requirement is $N \log k+O\left(N \frac{\log k}{\sqrt{\log N}}\right)$ bits of space per block. In the worst case every block has size $N_{\text {min }}$, and therefore the overall space is $n \log k+O\left(\frac{n \log k}{\sqrt{\log \log n}}\right)=n \log k+o(n \log k)$ bits of space.

Representing the Frontier of a Block. We could use a bit vector supporting rank and select to represent $F_{p}$, indicating with a 1 the nodes in the frontier. However, this would require $n+o(n)$ extra bits, exceeding our space limitation.

The frontier is instead represented by a conceptual increasingly-sorted array $\operatorname{Pre}_{p}\left[0 . . N_{c}\right]$ storing the preorders (within block $p$ ) of the nodes in the frontier of $p$ (i.e., those nodes having an inter-block pointer), except for $\operatorname{Pr}_{p}[0]=0$. Since 
the preorder of a node can change upon updates in $T_{p}$, we avoid the linear-time reconstruction of $\mathrm{Pre}_{p}$ by defining array $F_{p}\left[1 . . N_{c}\right]$, which stores the difference between consecutive preorders in $\operatorname{Pre}_{p}$, i.e. $F_{p}[i]=\operatorname{Pre}_{p}[i]-\operatorname{Pre}_{p}[i-1]$, for $i=1, \ldots, N_{c}$. Array $F_{p}$ is preprocessed with a data structure for searchable partial sums (see Section 2), denoting with $T_{F_{p}}$ the balanced tree representing the searchable partial sums. Since the total number of entries in arrays $F_{p}$ equals the number of blocks, the overall extra space requirement is $o(n)$ bits.

The preorder represented by a given $F_{p}[j]$ (i.e., the conceptual value $\operatorname{Pre}_{p}[j]$ ), is computed in $O(\log N)=O(\log k+\log \log n)$ time by $\operatorname{Sum}\left(F_{p}, j\right)$. Then, by using selectnode ${ }_{p}\left(\operatorname{Sum}\left(F_{p}, j\right)\right)$ we can get the DFuds position (and hence the representation) for that node in $O(\log k+\log \log n)$ time.

Representing Inter-block Pointers. In block $p$ we store the pointers to child blocks in the conceptual array $P T R_{p}\left[1 . . N_{c}\right]$, increasingly sorted according to the preorders of the nodes in the frontier of $p$. Since every pointer is associated to a node in the frontier of $p$, we store $P T R_{p}[i]$ along with $F_{p}[i]$ in the leaves of $T_{F_{p}}$.

For parent pointers, we store in each block $p$ a pointer to the representation of the root $r_{p}$ in the parent block $q$. As the DFUDS position of a node can change upon tree updates, we cannot store absolute parent pointers, which must be updated in linear worst-case time. Since $r_{p}$ lies within the frontier of $q$, we store in $p$ a pointer to the representation of $r_{p}$ in $F_{q}$ (i.e., a pointer to the leaf of $T_{F_{q}}$ corresponding to $r_{p}$ ). As a result, parent pointers are easily updated as needed, and we get the absolute parent pointer for block $p$ by first following the pointer to the leaf of $T_{F_{q}}$ representing $r_{p}$, and then computing Sum in $F_{q}$ up to that leaf.

The overall number of pointers equals the number of blocks in the structure, which is $O\left(n / \log ^{2} n\right)$. Thus, the overall space for pointers is $o(n)$ bits.

\subsection{Supporting Basic Operations}

We define the basic navigation operations for our dynamic data structure.

Operation child. To compute child $(x, i)$, if node $x$ is not a leaf, we use operation child $_{p}(x, i)$ inside block $p$, since each node is stored in the same block as its children. Operation child $(x, \alpha)$ is computed similarly using child $p(x, \alpha)$. If, on the other hand, node $x$ is a leaf, we check whether $x$ is a leaf of the whole tree (in whose case operation child gets undefined), or just a leaf of block $p$ (in whose case we have to follow a pointer to a child block).

We carry out that checking by computing the position $j=\operatorname{Search}\left(F_{p}\right.$, preorder $(x))$ in $F_{p}$ for the greatest preorder which is smaller or equal than the preorder of node $x$. Then we check whether the preorder represented by $F_{p}[j]$ (i.e., the value $\operatorname{Pre}_{p}[j]=\operatorname{Sum}\left(F_{p}, j\right)$ ) equals the preorder of node $x$. In such a case, $x$ is not a leaf of the whole tree, and then we have to follow the pointer $P T R_{p}[j]$ to get the child block $p^{\prime}$, to finally apply the corresponding child $p^{\prime}$ operation on the root of block $p^{\prime}$. Hence, operation $\operatorname{child}(x, i)$ is computed in $O(\log N)=$ 
$O(\log k+\log \log n)$ time, and operation child $(x, \alpha)$ takes $O\left(\log N\left(1+\frac{\log k}{\log \log N}\right)\right)=$ $O\left((\log k+\log \log n)\left(1+\frac{\log k}{\log (\log k+\log \log n)}\right)\right)$ worst-case time.

Operation parent $(x)$. If $x$ is not the root of block $p$ storing it, the operation is computed locally by using operation parent ${ }_{p}(x)$, since every non-root node is stored in the same block as its parent. Otherwise, we first follow the pointer to the parent block $q$, and then we compute the position of the representation of $x$ in the parent block $q$ as selectnode ${ }_{q}\left(\operatorname{Sum}\left(F_{q}, j\right)\right)$, assuming that block $p$ is the $j$-th child of block $q$. (As the parent pointer points to a leaf in $T_{F_{q}}$, we do not need to know $j$, but just to use the parent pointers in the searchable partial sum data structure for $F_{q}$.) Finally we apply parent ${ }_{q}$ on the representation of $x$ in block $q$, as we are sure that the parent of node $x$ is stored in $q$ (i.e., because node $x$ cannot be the root of block $q$, given the properties of our data structure). Operation parent is therefore computed in $O(\log N)=O(\log k+\log \log n)$ time.

Operation insert. Since we insert a new node $x$ in block $p$, we have to update the block accordingly. We first insert node $x$ in $T_{p}$, using operation insert ${ }_{p}$ of Lemma 2. Then, we insert in $S_{p}$ the new symbol $s$ labeling the new edge. Since the new node increases the preorders of some nodes in block $p$, every preorder in $F_{p}$ whose value is greater or equal to the preorder of $x$ must be increased: we look for position $j=\operatorname{Search}\left(F_{p}\right.$, $\left.\operatorname{preorder}(x)\right)$ in $F_{p}$ from where the preorders must be increased, and then we increase $F[j]$ by using operation Update. This automatically updates all preorders that have changed after the insertion of the new node. Notice that we are also automatically updating the parent pointers for the child blocks of $p$. In this way we avoid the (worst-case) linear update time of the frontier $F_{p}$. The insertion cost according to this procedure is $O((\log k+$ $\left.\log \log n)\left(1+\frac{\log k}{\log (\log k+\log \log n)}\right)\right)$ time, because of the time to update $S_{p}$.

Block Overflows. When inserting in a block $p$ of maximal size $N_{\max }$, we first divide the block $p$ into two blocks, both of size between $N_{\min }$ and $N_{\max }$, by selecting a node $z$ in block $p$ whose subtree will be reinserted in a new child block $p^{\prime}$ (including $z$ itself) and then will be deleted from $p$ (leaving node $z$ still in $p$ ). In this way $z$ is duplicated, since it is stored along with its children in $p^{\prime}$, and along with its siblings and parent in $p$, thus maintaining the properties of our data structure. Then, the insertion of node $x$ is carried out in the adequate block, either $p$ or $p^{\prime}$, without a new overflow since there is room for a new node in any of these blocks. When the subtree of node $z$ is reinserted in block $p^{\prime}$, we copy to $p^{\prime}$ the portions of arrays $F_{p}$ and $P T R_{p}$ corresponding to node $z$, via insertions in $F_{p^{\prime}}$ and $P T R_{p^{\prime}}$ and the corresponding deletions in $F_{p}$ and $P T R_{p}$.

After splitting $p$, we insert a new inter-block pointer in $P T R_{p}$ pointing to block $p^{\prime}$, and we add the preorder of node $z$ in $F_{p}$, at the corresponding position (marking that $z$ lies now within the frontier of $p$ ). We add also a parent pointer in $p^{\prime}$, pointing to the leaf corresponding to $z$ in the tree $T_{F_{p}}$ representing $F_{p}$. In this simple way we keep up-to-date all of the parent pointers for the children of $p$, since the other pointers do not change after adding a new child block to $p$. 
In order to amortize the insertion cost, the overall reinsertion process must be carried out in time proportional to the size of the reinserted tree. The work on $T_{p}, S_{p}, F_{p}$, and pointers can be done in this time, by using the corresponding insert and delete operations on them. We must be careful, however, with the selection of node $z$, since naively this would take linear time. Thus, we define a list of candidate nodes $C_{p}$ for every block $p$, storing the local preorders of candidate nodes to be reinserted upon overflow. We represent $C_{p}$ in the same way as $F_{p}$, in differential form and using a searchable partial sum data structure.

To maintain $C_{p}$ we must dynamically sample some nodes of $T_{p}$ such that, every time we need to split $p$, there is at least a candidate subtree to be reinserted in the new child block. We must also ensure that the overall space for the $C_{p}$ data structures is $o(n)$ bits, so we cannot maintain too many candidates.

Thus, every time we descend in the tree we maintain the last node $z$ in block $p$ such that subtreesize ${ }_{p}(z) \geqslant N_{\min }$ holds. When we find the insertion point of the new node $x$, say at block $p$, before adding $z$ to $C_{p}$ we first perform $p_{1}=\operatorname{Search}\left(C_{p}, \operatorname{preorder}_{p}(z)\right)$, and then $p_{2}=\operatorname{Search}\left(C_{p}, \operatorname{preorder}_{p}(z)+\right.$ subtreesize $\left._{p}(z)\right)$. Then, we add $z$ to $C_{p}$ whenever: $(\mathbf{1}) z$ is not the root of block $p$; and $(\mathbf{2})$ it holds that $p_{1}=p_{2}$, which means that there is no other candidate in the subtree of $z$. If in the descent we find a candidate node $z^{\prime}$ which is an ancestor of $z$, then after inserting $z$ to $C_{p}$ we delete $z^{\prime}$ from $C_{p}$. In this way we keep the lowest possible candidates, avoiding that the subtree of a candidate becomes so large, which would not guarantee a fair partition into two blocks of size between $N_{\min }$ and $N_{\max }$ upon overflow.

As a result we ensure that the local subtree size of every candidate is at least $N_{\min }$, and also that given a candidate node $z$, there are no candidate nodes in the subtree of $z$. Thus, we have a candidate node out of (at least) $N_{\min }$ nodes, and hence the total space to manage the candidates is $o(n)$. We are also ensuring that every time a block becomes full we have at least one candite node in $C_{p}$ to be reinserted, because there were sufficient insertions in $p$ (to become full) in order to find at least a candidate in it. This is because of the maximum block size $N_{\text {max }}=\Theta\left(k \log ^{2} n\right)$ that we have chosen: this ensures that whenever a block becomes full, at least one of the children of the block root has size at least $N_{\text {min }}$.

The reinsertion cost is proportional to the size of the reinserted subtree; since we have already paid to insert these nodes for the first time, the insertion cost is $O\left(\log N\left(1+\frac{\log k}{\log \log N}\right)\right)=O\left((\log k+\log \log n)\left(1+\frac{\log k}{\log (\log k+\log \log n)}\right)\right)$ amortized.

Operation delete. To delete a node $x$ in block $p$ we update the data structure by using operation delete $e_{p}$. After deleting $x$, we check whether there is a candidate node $z$ in $C_{p}$ which is ancestor of $x$ and whose subtree becomes smaller than $N_{\min }$ after deleting $x$. As there is at most one ancestor of $x$ in $C_{p}, z$ can be found as the node represented by $C_{p}\left[\operatorname{Search}\left(C_{p}\right.\right.$, $\left.\left.\operatorname{preorder}_{p}(x)\right)-1\right]$; the subtraction comes from the fact that with the search in $C_{p}$ we find a candidate which is next (in preorder) to $z$ in $C_{p}$. After deleting $z$ from $C_{p}$, we try to insert in $C_{p}$ the last node $z^{\prime}$ found in the descent (before the deletion) whose subtree size is greater or equal to $N_{\text {min }}$, following the same policies as for operation insert. 
If we delete $x$ from a block $p$ of size $N_{\text {min }}$, then a block underflow occurs. In such a case, we find the representation of the block root $r_{p}$ in the parent block $q$, by using the corresponding parent pointer. From that node we reinsert in $q$ all of the nodes of block $p$. Note that in the worst case there will be only one block overflow in $q$ when reinserting, since block $p$ has less than $N_{\text {min }}$ nodes, and after an overflow in $q$ there will be room for at least $N_{\min }$ new nodes. If $p$ is not a leaf in the tree of blocks, we reinsert the frontier of $p$ within the frontier of $q$.

Managing Dynamic Memory. The model of memory allocation is a fundamental issue of succinct dynamic data structures, since we must be able to manage the dynamic memory fast and without requiring so much memory space due to memory fragmentation. We assume a standard model where the memory is regarded as an array, with words numbered 0 to $2^{w}-1$. The space usage of an algorithm at a given time is the highest memory word currently in use by the algorithm. This corresponds to the so-called $\mathcal{M}_{B}$ memory model [21].

We manage the memory of every tree block separately, each in a "contiguous" memory space. However, tree blocks are dynamic and therefore this memory space must grow and shrink accordingly. If we use an Extendible Array (EA) [6] to manage the memory of a given block, we end up with a collection of at most $O\left(n / \log ^{2} n\right)$ EAs, which must be maintained under the operations: create, which creates a new empty EA in the collection; destroy, which destroys an EA from the collection; $\operatorname{grow}(A)$, which increases the size of array $A$ by one; $\operatorname{shrink}(A)$, which shrinks the size of array $A$ by one; and access $(A, i)$, which access the $i$-th item in array $A$. Raman and Rao [21] show how operation access can be supported in $O(1)$ worst-case time, create, grow and shrink in $O(1)$ amortized time, and destroy in $O\left(s^{\prime} / w\right)$ time, where $s^{\prime}$ is the nominal size (in bits) of array $A$ to be destroyed. The space requirement for the whole collection is $s+O\left(a^{*} w+\sqrt{s a^{*} w}\right)$ bits, where $a^{*}$ is the maximum number of EAs that ever existed simultaneously in the collection, and $s$ is the nominal size of the collection.

To simplify the analysis we store every part of a block in different collection of EAs (i.e., we have a collection for $T_{p} \mathrm{~s}$, a collection for $S_{p} \mathrm{~s}$, and so on). The memory for $S_{p}$ and $T_{p}$ inside the corresponding EA is managed as in the original works $[11,16]$. For the case of $F_{p}, C_{p}$, etc., we manage the corresponding EA by using standard techniques to allocate and free dynamic memory. Thus, we use operation grow on the corresponding EA every time we insert a node in the tree, operation shrink when we delete a node, and operation create upon block overflows, all of them in $O(1)$ amortized time. Operation destroy, on the other hand, is used upon block underflows. Consider the EA collection storing $S_{p}$ for every block $p$ of the tree. The block $p^{\prime}$ which underflows has size less than $\Theta\left(\log ^{2} n\right)$, and thus the nominal size for the EA storing $S_{p^{\prime}}$ is less than $\Theta\left(\log ^{2} n \log k\right)$ bits. Therefore operation destroy takes less than $\Theta(\log n \log k)$ time, which is negligible since we have to reinsert all of the nodes of $p^{\prime}$ in the parent block, at a higher cost. The EAs storing the remaining parts of $p^{\prime}$ can be destroyed even faster. 
For the space analysis, it is important to note that every time $\log n$ changes, the tree must be rebuilt from scratch to adapt these changes. This also involves rebuilding the data structures needed to maintain the collections of EAs. The amortized cost of update operations over the tree still remains the same. Let $n^{\prime}$ be the maximum number of nodes that ever existed in the tree since the last reconstruction (i.e., the last change of $\log n$ ). As reconstructions occur when $n$ is a power of two, then both $n$ and $n^{\prime}$ lie between (the same) two consecutive powers of two, and thus we can prove that $n \leqslant n^{\prime} \leqslant 2 n$ holds, which means $n^{\prime}=\Theta(n)$. Thus, we can conclude that the maximum number of EAs that we can have between reconstructions is $a^{*}=O\left(n / \log ^{2} n\right)$.

The nominal size of the EA collection for $T_{p} \mathrm{~s}$ is $2 n+o(n)$ bits. Then, this collection requires $2 n+o(n)+O\left(\frac{n}{\log n}+\frac{n}{\sqrt{\log n}}\right)=2 n+o(n)$ bits of space [21]. The nominal size of the collection for $S_{p} \mathrm{~s}$ is $n \log k+o(n \log k)$, and thus we have $n \log k+o(n \log k)+O\left(\frac{n}{\log n}+n \sqrt{\log k / \log n}\right)=n \log k+o(n \log k)$ bits overall.

Therefore we have proved:

Theorem 1. There exists a representation for dynamic $k$-ary trees using $2 n+$ $n \log k+O\left(\frac{n \log k}{\sqrt{\log \log n}}\right)$ bits of space supporting operations parent and child $(x, i)$ in $O(\log k+\log \log n)$ worst-case time, operation child $(x, \alpha)$ in $O((\log k+\log \log n)(1+$ $\left.\left.\frac{\log k}{\log (\log k+\log \log n)}\right)\right)$ worst-case time, and operations insert and delete in $O((\log k+$ $\left.\log \log n)\left(1+\frac{\log k}{\log (\log k+\log \log n)}\right)\right)$ amortized time.

In the case of binary non-labeled trees $[19,21]$ we have:

Corollary 1. There exists a representation for dynamic binary trees using $2 n+$ $o(n)$ bits of space and supporting operations parent and child $(x, i)$ in $O(\log \log n)$ worst-case time, and operations insert and delete in $O(\log \log n)$ amortized time.

Thus we improve the $O\left((\log \log n)^{1+\epsilon}\right)$ update time of [21], but at the price of more expensive navigations ([21] provides $O(1)$ time for these operations).

We leave the definition of more involved operations (like subtreesize, depth, etc.) for the full paper. Most of them are supported in $O(\log k+\log \log n)$ time.

\section{Conclusions and Further Works}

We have defined a succinct representation for dynamic $k$-ary trees (or tries) of $n$ nodes, requiring $2 n+n \log k+o(n \log k)$ bits of space and supporting navigation operations in $O(\log k+\log \log n)$ time, as well as insertion and deletion of leaves in $O\left((\log k+\log \log n)\left(1+\frac{\log k}{\log (\log k+\log \log n)}\right)\right)$ amortized time. Our representation is able to take advantage of asymptotically smaller values of $k$, thus improving the $O(\log n)$ time achieved by alternative representation of Lemma 2 whenever $\log k=o(\log n)$, which covers many interesting applications in practice.

An interesting future work is to reduce the extra space of $O\left(\frac{n \log k}{\sqrt{\log \log n}}\right)=$ $o(n \log k)$ bits needed by our representation. This comes from the data structure of [11] used to represent $S_{p}$ in each block. Also, it would be interesting to reduce 
the time of the operations (e.g., to $O\left(\frac{\log \log n}{\log \log \log n}\right)$ time) in the case of small alphabets, e.g. $k=O(\operatorname{polylog}(n))$ (a particular case is that of binary trees).

Acknowledgments. We thank Jérémy Barbay for proofreading this paper.

\section{References}

1. Apostolico, A.: The myriad virtues of subword trees. In: Combinatorial Algorithms on Words. NATO ISI Series, Springer-Verlag (1985) 85-96

2. Arroyuelo, D., Navarro, G.: A Lempel-Ziv text index on secondary storage. In: Proc. CPM. LNCS 4580 (2007) 83-94

3. Arroyuelo, D., Navarro, G.: Space-efficient construction of LZ-index. In: Proc. ISAAC. LNCS 3827 (2005) 1143-1152

4. Barbay, J., He, M., Munro, J.I., Rao, S.S.: Succinct indexes for strings, binary relations and multi-labeled trees. In: Proc. SODA. (2007) 680-689

5. Benoit, D., Demaine, E., Munro, J.I., Raman, R., Raman, V., Rao, S.S.: Representing trees of higher degree. Algorithmica 43(4) (2005) 275-292

6. Brodnik, A., Carlsson, S., Demaine, E., Munro, J.I., Sedgewick, R.: Resizable arrays in optimal time and space. In: Proc. WADS. LNCS 1663 (1999) 37-48

7. Chan, H.L., Hon, W.K., Lam, T.W., Sadakane, K.: Compressed indexes for dynamic text collections. ACM TALG 3(2) (2007) article 21

8. Ferragina, P., Luccio, F., Manzini, G., Muthukrishnan, S.: Structuring labeled trees for optimal succinctness, and beyond. In: Proc. FOCS. (2005) 184-196

9. Ferragina, P., Manzini, G., Mäkinen, V., Navarro, G.: Compressed representations of sequences and full-text indexes. ACM TALG 3(2) (2007) article 20

10. Geary, R., Raman, R., Raman, V.: Succinct ordinal trees with level-ancestor queries. In: Proc. SODA. (2004) 1-10

11. González, R., Navarro, G.: Improved dynamic rank-select entropy-bound structures. In: Proc. LATIN. (2008) To appear.

12. Hon, W.K., Sadakane, K., Sung, W.K.: Succinct data structures for searchable partial sums. In: Proc. ISAAC. LNCS 2906 (2003) 505-516

13. Jacobson, G.: Space-efficient static trees and graphs. In: Proc. FOCS. (1989) 549-554

14. Jansson, J., Sadakane, K., Sung, W.K.: Ultra-succinct representation of ordered trees. In: Proc. SODA. (2007) 575-584

15. Katajainen, J., Mäkinen, E.: Tree compression and optimization with applications. Int. J. Found. Comput. Sci. 1(4) (1990) 425-448

16. Mäkinen, V., Navarro, G.: Dynamic entropy-compressed sequences and full-text indexes. ACM TALG (2007) To appear.

17. Manzini, G.: An analysis of the Burrows-Wheeler transform. Journal of the ACM 48(3) (2001) 407-430

18. Munro, J.I., Raman, V.: Succinct representation of balanced parentheses and static trees. SIAM Journal on Computing 31(3) (2001) 762-776

19. Munro, J.I., Raman, V., Storm, A.: Representing dynamic binary trees succinctly. In: Proc. SODA. (2001) 529-536

20. Raman, R., Raman, V., Rao, S.S.: Succinct indexable dictionaries with applications to encoding $k$-ary trees and multisets. In: Proc. SODA. (2002) 233-242

21. Raman, R., Rao, S.S.: Succinct dynamic dictionaries and trees. In: Proc. ICALP. LNCS 2719 (2003) 357-368 